\title{
THE STRUCTURE AND ASYMPTOTIC BEHAVIOR OF POLYNOMIALLY COMPACT OPERATORS ${ }^{1}$
}

\author{
FRANK GILFEATHER
}

Abstract. A. R. Bernstein and A. Robinson proved that every polynomially compact operator in Hilbert space has nontrivial invariant subspaces. This paper gives a structure theorem for these operators. We show that a polynomially compact operator is the finite sum of translates of operators which have the property that a finite power of the operator is compact. Furthermore, the spectrum of polynomially compact operators is completely described. Conditions are given to determine the weak and strong asymptotic behavior of a polynomially compact contraction in Hilbert space.

We say that a bounded operator $A$ on a complex Banach space $B$ is polynomially compact if there is a nonzero complex polynomial $p(z)$ such that the operator $p(A)$ is compact. In $\$ 1$ we shall give a structure theorem for these operators which reduces their study to the study of power compact operators. We shall show that a polynomially compact operator is just the finite sum of translates of operators which have the property that a finite power of the operator is compact. In case the space is a Hilbert space and the operator is a normal operator, then it is just the direct sum of translates of compact normal operators and hence its complete structure can be determined. Furthermore, we can exactly describe the spectrum of these operators. Our structure theorems, besides describing the spectrum of such operators, will have several applications which we present in $\$ 2$.

It is immediate from the structure theorem that the existence of nontrivial invariant subspaces follows directly from the theorem in [2] for Hilbert spaces and from the theorem in [7] for Banach spaces. In the latter case this corollary was not stated. Furthermore, we can give necessary and sufficient conditions on the spectrum of a polynomially compact contraction on Hilbert space so that the operator

Received by the editors August 7, 1969.

A MS Subject Classifications. Primary 4740; Secondary 4730-4735, 4745.

Key Words and Phrases. Polynomially compact operator, asymptotic behavior, structure theorem, determination of spectrum, invariant subspaces, hyponormal operator, normal operator.

1 This work is part of the author's Ph.D. dissertation at the University of California, Irvine, directed by Professor Noboru Suzuki. The author was a N.S.F. trainee during this time. 
is weak and strong asymptotically convergent. Using the result developed by $F$. Browder and W. Petryshyn [4], we can then completely solve the operator equation $(I-T) x=y$ for such operators. And, in fact, if $\sigma_{p}(T) \cap S^{1}=\varnothing\left(S^{1}=\{z \in C:|z|=1\}\right)$ we can solve the resolvent equation $(\lambda I-T) x=y$ for $|\lambda|=1$.

An operator on a Hilbert space $H$ is called hyponormal in case $\left\|T^{*} x\right\| \leqq\|T x\|$ for all $x \in H$ or equivalently if $T^{*} T-T T^{*} \geqq 0$. S. Berberian [3] asked whether every compact hyponormal operator is normal. T. Andö [1] and J. Stampfli [10] have given an affirmative answer to this question. Using the structure theorem or the spectral properties of a polynomially compact operator we can show that every polynomially compact hyponormal operator is in fact normal and hence has a structure given by Theorem 2 .

In this paper all operators are bounded. The notation $S^{1}$ is used to denote the circumference of the unit circle in $C$, that is, $S^{1}=\{\lambda|| \lambda \mid$ $=1\}$.

1. In this section we shall first prove the structure theorem for polynomially compact operators on a Banach space $\mathfrak{X}$. Then we shall give the more complete structure theorem for a polynomially compact normal operator on Hilbert space.

Let $B$ be a polynomially compact operator. Because of the well ordering of the positive integers there exists a nonzero polynomial $p(z)$ of least degree such that $p(B)$ is compact. In fact, $p(z)$ can be made unique by specifying the value of the leading coefficient, that is, the coefficient on the term with largest degree.

Definition. For a polynomially compact operator $B$, the nonzero polynomial $p(z)$, of least degree and leading coefficient 1 such that $p(B)$ is compact, will be called the minimal polynomial of $B$.

Now we state and prove the main structure theorem.

THEOREM 1. Let $B$ be a polynomially compact operator with minimal polynomial $p(z)=\left(z-\lambda_{1}\right)^{n_{1}} \cdots\left(z-\lambda_{k}\right)^{n_{k}}$. Then the Banach space $\mathfrak{X}$ is decomposed into the direct sum $\mathfrak{X}=\mathfrak{X}_{1} \oplus \cdots \oplus \mathfrak{X}_{k}$ and $B=B_{1} \oplus \cdots$ $\oplus B_{k}$, where $B_{i}$ is the restriction of $B$ to $\mathfrak{X}_{i}$. The operators $\left(B_{i}-\lambda_{i} I_{i}\right)^{n_{i}}$ are all compact. The spectrum of $B$ consists of countably many points with $\left\{\lambda_{1}, \cdots, \lambda_{k}\right\}$ as the only possible limit points and such that all but possibly $\left\{\lambda_{1}, \cdots, \lambda_{k}\right\}$ are eigenvalues with finite dimensional generalized eigenspaces. Each point $\lambda_{i} \in\left\{\lambda_{1}, \cdots, \lambda_{k}\right\}$ is either the limit of eigenvalues of $B$ or else $\left(B_{i}-\lambda_{i} I_{i}\right)$ is quasi nilpotent with $\mathfrak{X}_{i}$ infinite dimensional.

Proof. If $\lambda_{i}$ is a root of $p(z)$ and $\lambda_{i} \notin \sigma(B)$, then let $q(z)=$ $\left(z-\lambda_{i}\right)^{-1} p(z)$. Clearly, $q(B)$ is also compact and the order of 
$q(z)<$ order of $p(z)$, contradicting the minimality of $p(z)$. Thus $\lambda_{i}$ $\in \sigma(B)$ for all roots $\lambda_{i}$ of $p(z)$. Since $\sigma(p(B))=p(\sigma(B))$, by the Spectral Mapping Theorem and because of the fact that $\sigma(p(B))$ is finite or countable, we obtain that $\sigma(B)$ must be finite or countable. Since zero is the only possible accumulation point of $\sigma(p(B))$ and $p$ is continuous, the only possible accumulation points of $\sigma(B)$ are the roots of $p(z)$. We shall now show that if $\lambda$ is an isolated point of $\sigma(B)$ such that $p(\lambda) \neq 0$, then $\lambda$ is an eigenvalue of $B$ with finite dimensional generalized eigenspace. We can find a suitable domain $\delta$ in $C$ (a domain whose boundary is a rectifiable Jordan curve) such that $\lambda \in \delta$ and $\bar{\delta} \cap \sigma(B)=\{\lambda\}$. Let $E(\delta)=\int_{\partial(\delta)}(B-\lambda I)^{-1} d \lambda$, then it is known that $\mathfrak{X}_{\lambda}=E(\delta) \mathfrak{X}$ is an invariant subspace of $B$ such that $\sigma\left(B / \mathfrak{X}_{\lambda}\right)=\{\lambda\}$. Since $p(\lambda) \neq 0$, we have $0 \notin \sigma\left(p\left(B / \mathfrak{X}_{\lambda}\right)\right)$ so $p\left(B / \mathfrak{X}_{\lambda}\right)$ is invertible and completely continuous. This is true only if $\mathfrak{X}_{\lambda}$ is finite dimensional. Since $\{\lambda\}=\sigma\left(B / \mathfrak{X}_{\lambda}\right)$ and $\mathfrak{X}_{\lambda}$ is a finite dimensional space, we have that $\mathfrak{X}_{\lambda}=\left\{x \mid(B-\lambda I)^{n} x=0\right\}$ for $n$ large enough. Hence each $\lambda \in \sigma(B)$ such that $p(\lambda) \neq 0$ is an eigenvalue and its generalized eigenspace is finite dimensional.

Now let $\lambda \in \sigma(B), p(\lambda)=0$ and such that $\lambda$ is an isolated point in $\sigma(B)$. As above, we can find suitable domains $\delta$ and $\tau$ such that $\bar{\delta} \cap \bar{\tau}=\varnothing, \delta \cup \tau \supset \sigma(B), \lambda \in \delta$ and $\bar{\delta} \cap \sigma(B)=\{\lambda\}$. If $\mathfrak{X}_{\delta}=E(\delta) \mathfrak{X}$ and $\mathfrak{X}_{\tau}=E(\tau) \mathfrak{X}$, we have that $\mathfrak{X}=\mathfrak{X}_{\delta} \oplus \mathfrak{X}_{\tau}$ and $B=B_{\delta} \oplus B_{\tau}$ where $B_{\delta}$ (resp. $\tau$ ) is $B \mid \mathfrak{X}_{\delta}$ (resp. $\tau$ ). Also $\sigma\left(B_{\delta}\right)=\{\lambda\}$ and $\lambda \notin \sigma\left(B_{\tau}\right)$. Thus if $I_{\delta}$ (resp. $\tau$ ) is $I \mid \mathfrak{X}_{\delta}$ (resp. $\tau$ ), then $\left(B_{\tau}-\lambda I_{\tau}\right)^{-1} p\left(B_{\tau}\right)$ is compact. In order that $p(z)$ be the minimal polynomial of $B$ it is necessary that $\mathfrak{X}_{\delta}$ be infinite dimensional. Therefore $\left(B_{\delta}-\lambda I_{\delta}\right)$ is quasi nilpotent and the generalized eigenspace for $\lambda$ is infinite dimensional.

Therefore if $p(\lambda)=0$, we have shown that $\lambda \in \sigma(B)$ and is either the limit of points in $\sigma(B)$ each having a finite dimensional generalized eigenspace or $(B-\lambda I) / \mathfrak{X}_{\delta}$ is quasi nilpotent for a suitably chosen domain $\delta$. Also if $p(\lambda) \neq 0$ and $\lambda \in \sigma(B)$ we have that $\lambda$ is an eigenvalue with a finite dimensional generalized eigenspace.

Now by induction we may construct open sets $\left\{\delta_{i}\right\}_{i=1}^{k}$ in the complex plane, corresponding to the set $\left\{\lambda_{1}, \cdots, \lambda_{k}\right\}$ of roots of $p(z)$, so that:

(i) $\lambda_{i} \in \delta_{i}$ for $i=1, \cdots, k$,

(ii) the boundaries of each $\delta_{i}$ is a rectifiable Jordan curve,

(iii) $\bar{\delta}_{i} \cap \bar{\delta}_{j}=\varnothing$ if $i \neq j$,

(iv) if $\lambda_{i}$ is an isolated point of $\sigma(B)$, then $\delta_{i} \cap \sigma(B)=\left\{\lambda_{i}\right\}$ and

(v) $\sigma(B) \subset \bigcup_{i=1}^{k} \delta_{i}$.

Let $E\left(\delta_{i}\right)=\int_{\partial\left(\delta_{i}\right)}(B-\lambda I)^{-1} d \lambda$, then $E\left(\delta_{i}\right) \mathfrak{X} \equiv \mathfrak{X}_{i}$ is an invariant subspace for $B$. Let $B_{i}$ be the restriction of $B$ to $\mathfrak{X}_{i}$ and $I_{i}=I / \mathfrak{X}_{i}$. By the 
properties of the operators $E\left(\delta_{i}\right)$ and the construction of the sets $\left\{\delta_{i}\right\}$, we have that $\mathfrak{X}=\mathfrak{X}_{1} \oplus \cdots \oplus \mathfrak{X}_{k}, \quad B=B_{1} \oplus \cdots \oplus B_{k}$ and $\sigma\left(B / \mathfrak{X}_{i}\right)=\sigma(B) \cap \delta_{i}$. Finally, let us show that $\left(B_{i}-\lambda_{i} I_{i}\right)^{n_{i}}$ is a compact operator. Since $B_{i}-\lambda_{j} I_{i}$ is invertible for each $j \neq i$, we have that $\left(z-\lambda_{i}\right)^{n_{i}}=\prod^{j \neq i}\left(z-\lambda_{j}\right)^{-n_{j}} p(z)$ is a polynomial which makes $B_{i}$ compact.

As an illustration of this decomposition let us consider the case of a normal operator $B$ on a Hilbert space $H$. If $B$ is also polynomially compact we can obtain the following structure theorem for $B$.

THEOREM 2. Let $B$ be a polynomially compact normal operator on a Hilbert space $H$. Let $p(z)=\left(z-\lambda_{1}\right)^{n_{1}} \cdots\left(z-\lambda_{k}\right)^{n_{k}}$ be the minimal polynomial of $B$. Then $\sigma(B)$ consists of countably many points such that all but possibly $\left\{\lambda_{1}, \cdots, \lambda_{k}\right\}$ are eigenvalues with finite dimensional eigenspaces. The rest, $\left\{\lambda_{1}, \cdots, \lambda_{k}\right\}$, are of either of the following types:

(a) limit points of eigenvalues,

(b) isolated eigenvalues with infinite dimensional eigenspaces. Then $B$ is decomposed into the orthogonal direct sum

$$
B=B_{1} \oplus \cdots \oplus B_{k} \quad \text { where } H=H_{1} \oplus \cdots \oplus H_{k}
$$

and $B_{i}-\lambda_{i} I_{i}$, which is $B-\lambda_{i} I$ reduced to $H_{i}$, is compact and, in fact, if $\lambda_{i}$ is a root of type (b), then $B_{i}=\lambda_{i} I_{i}$.

Proof. This theorem follows from the previous theorem and certain facts about Hilbert spaces and normal operators. If we make the decomposition as in Theorem 1 and call the invariant spaces $H_{i}$ instead of $\mathfrak{X}_{i}$, we have that each $H_{i}$ reduces $B$ and, furthermore, $H_{i} \perp H_{j}$ if $i \neq j$. Thus we can decompose $H$ into the orthogonal direct sum $H=H_{1} \oplus \cdots \oplus H_{k}$ and $B=B_{1} \oplus \cdots \oplus B_{k}$. Also from Theorem 1 we have that $\left(B_{i}-\lambda_{i} I_{i}\right)^{n_{i}}$ is compact. Since $B_{i}-\lambda_{i} I_{i}$ is normal, we can conclude that $B_{i}-\lambda_{i} I_{i}$ is compact. If $\lambda_{i}$ is isolated, then $B_{i}-\lambda_{i} I_{i}$ is a quasi nilpotent normal operator and hence it is zero. Therefore, if $\lambda_{i}$ is a root of $p(z)$ of type (b), then $B_{i}=\lambda_{i} I_{i}$ and since $H_{i}$ must be infinite dimensional, then $\lambda$ has an infinite dimensional eigenspace.

2. In this section we shall present several applications of the structure theorems of $\S 1$.

Nontrivial invariant subspaces for polynomially compact operators on Hilbert spaces were first found by A. R. Bernstein and A. Robinson. P. R. Halmos [8] simplified their proof and W. Arveson and J. Feldman [2] gave a theorem from which the existence of invariant subspaces of polynomially compact operators on Hilbert space follows. Finally, T. A. Gillespie [7] has given a Banach space version 
of the result in [2]. It follows from [7] that a polynomially compact operator on Banach space has nontrivial invariant subspaces.

Proposition 1. If $B$ is a polynomially compact operator on a Banach space $\mathfrak{X}$ of dimension greater than 1 , then there exists nontrivial invariant subspaces for $B$.

Proof. By Theorem 1 we need only consider a proof for operators $B$ which are quasi nilpotent and power compact. Clearly if $B \neq 0$ and $B^{n}=0$ for some $n$, then $\{x \mid B x=0\}$ is a nontrivial invariant subspace of $B$. If $B^{n}$ is compact and nonzero, then by the theorem given in [7], $B$ has nontrivial invariant subspaces.

Using a result of B. Sz.-Nagy and C. Foiaş we can determine a necessary and sufficient spectral condition for a contraction operator $T$ on a Hilbert space $H$ to be strong asymptotically convergent, that is, $\left\{T^{n}\right\}$ converges in the strong operator topology.

Proposition 2. Let $T$ be a contraction operator on a Hilbert space $H$ for which $\sigma(T) \cap S^{1}$ is countable. Then $T$ is strong asymptotically convergent if and only if $\sigma_{p}(T) \cap S^{1} \subset\{1\}$.

Proof. Let $H=H_{0} \oplus H_{1}$ be the decomposition of $H$ such that $T=T_{0} \oplus T_{1}$, the corresponding decomposition of $T$, is the decomposition of $T$ into unitary and completely nonunitary parts [12, Theorem I.3.2]. ${ }^{2}$ If we assume that $\sigma_{p}(T) \cap S^{1} \subset\{1\}$, then $\sigma_{p}\left(T_{0}\right)$ $C\{1\}$. Because $\sigma\left(T_{0}\right)$ is a subset of the countable set $\sigma(T) \cap S^{1}$, it is also countable. Since $T_{0}$ is normal every isolated point of $\sigma\left(T_{0}\right)$ is an eigenvalue, but $\sigma_{p}\left(T_{0}\right) \subset\{1\}$ so that 1 is the only possible isolated point of $\sigma\left(T_{0}\right)$. If 1 is not an isolated point of $\sigma\left(T_{0}\right)$, then $\sigma\left(T_{0}\right)$ is a countable perfect set, that is, a closed set where every point is a limit point. Since a complete metric space has no nonempty countable perfect sets, we may conclude that $\sigma\left(T_{0}\right)=\varnothing$ and $H_{0}=\{0\}[9, \mathrm{p} .72]$. If however 1 is an isolated point of $\sigma\left(T_{0}\right)$, then the same argument applied to $\sigma\left(T_{0}\right)-\{1\}$ implies that $\sigma\left(T_{0}\right)=\{1\}$. Because $T_{0}$ is unitary, $T_{0}-I \mid H_{0}$ is quasi nilpotent and normal and therefore must be zero. Thus $T_{0}=I \mid H_{0}$ and $T_{0}^{n}$ converges uniformly and therefore $T$ is strong asymptotically convergent on $H_{0}$.

We have $\sigma\left(T_{1}\right) \cap S^{1} \subset \sigma(T) \cap S^{1}$, so that $\sigma\left(T_{1}\right) \cap S^{1}$ is countable and thus the Lebesgue measure of $\sigma\left(T_{1}\right) \cap S^{1}$ is zero. Now from Proposition II.6.7 [12] we have that $T_{1}^{n} \rightarrow 0$ in the strong topology. Thus, $T^{n}=T_{0}^{n} \oplus T_{1}^{n} \rightarrow P$ strongly, where $P$ is the projection on $H_{0}$.

${ }^{2}$ An operator $T$ is said to be completely nonunitary (resp. selfadjoint, normal), if $T$ does not have a nonzero reducing subspace $\mathfrak{T}$ such that $T / \mathfrak{T}$ is unitary (resp. selfadjcint, normal). 
Conversely if $T^{n}$ converges strongly it is necessary that $\lambda$ is not an eigenvalue of $T$ if $|\lambda| \geqq 1$ and $\lambda \neq 1$.

From this proposition and Theorem 1 we can give spectral conditions for a polynomially compact contraction operator on a Hilbert space to converge asymptotically.

Corollary 1. Let $B$ be a polynomially compact contraction operator on Hilbert space. Then $B$ is strong asymptotically convergent if and only if $\sigma_{p}(B) \cap S^{1} \subset\{1\}$.

For certain classes of operators on Hilbert space the notion of strong asymptotic convergence coincides with that of weak asymptotic convergence, that is, the weak convergence of the iterates of the operator. It is known that whenever $T$ is a selfadjoint or compact operator, then $\left\{T^{n}\right\}$ converges strongly if $\left\{T^{n}\right\}$ converges weakly $[\mathbf{5}$, Lemmas 3 and 5]. Using Theorem 1 it can be shown that polynomially compact operators also have this property [6, Lemma I.3.11].

Proposition 3. Let $T$ be a polynomially compact operator on Hilbert space, then $\left\{T^{n}\right\}$ converges strongly if and only if $\left\{T^{n}\right\}$ converges weakly.

F. E. Browder and W. V. Petryshyn have shown that if $T$ is strong asymptotically convergent, the operational equation $(I-T) x=y$ has solutions [4]. By a solution it is meant that whenever $y$ is an element of the range of $I-T$, we can generate a sequence $\left\{x_{n}\right\}$ such that $x_{n} \rightarrow x$ and $(I-T) x=y$. In fact, the sequence $\left\{x_{n}\right\}$ can be defined by

$$
x_{n}=T^{n} x_{0}+\sum_{i=0}^{n-1} T^{i} y
$$

where $x_{0}$ is any initial approximation. It is easy to show that if $T^{n} \rightarrow Q$, then all solutions of $(I-T) x=y$ are of the form $x_{0}+Q(z)$ where $x_{0}$ is one solution and $z$ any vector in the space.

Let $B$ be a polynomially compact contraction operator on a Hilbert space $H$. If $\sigma_{p}(B) \cap S^{1} \subset\{1\}$, then by Corollary $1,\left\{B^{n}\right\}$ converges so that solutions of $I-B$ can be determined. If $\sigma_{p}(B) \cap S^{1}=\varnothing$, then $\left\{(\bar{\lambda} B)^{n}\right\}$ converges for $|\lambda|=1$ and solutions of $\lambda I-B$ can be determined. In case $\sigma_{p}(B) \cap S^{1}=\varnothing$, these solutions are unique. Thus $\left\{\sum_{i=0}^{n} \bar{\lambda}^{i} T^{i} y\right\}$ converges to a solution of $(\lambda I-T) x=y$, whenever $y$ is in the range of $\lambda I-T$.

Several authors have tried to determine which hyponormal operators on Hilbert space are normal [1], [10], and [11]. We shall show that any hyponormal operator with a countable spectrum is normal. This will generalize Corollary 2 of [10]. 
Proposition 4. Let T be a hyponormal operator on a Hilbert space $H$. If $\sigma(T)$ is countable, then $T$ is normal.

Proof. Let $T=T_{0} \oplus T_{1}$ be the canonical decomposition of $T$ into normal and completely nonnormal parts. Let $H=H_{0} \oplus H_{1}$ be the corresponding decomposition of $H$, so that $T_{1}$ is the normal part of $T$. Since $\sigma\left(T_{0}\right) \subset \sigma(T)$ it is also countable. Now $T_{0}$ is hyponormal and completely nonnormal, that is, $T_{0}$ has no reducing subspace on which it is normal. Thus by Theorem 2 in [10], $\sigma\left(T_{0}\right)$ has no isolated points. Since $\sigma\left(T_{0}\right)$ is also countable and closed, it is a countable perfect set and we may conclude that $\sigma\left(T_{0}\right)=\varnothing\left[9\right.$, p. 72]. Therefore $H_{0}=\{0\}$, $T=T_{1}$ and $T$ is normal.

Using the spectral conditions given in Theorem 1 and Proposition 4 , we may conclude that every polynomially compact hyponormal operator is normal.

COROLlaRy 2. If $T$ is a hyponormal polynomially compact operator, then $T$ is normal.

Thus using Theorem 2 we have the complete structure of hyponormal polynomially compact operators.

ADDED IN PROOF. Theorems 1 and 2 need a slight modification in the case that each root $\lambda_{i}$ of the minimal polynomial of $A$ is an isolated point of $\sigma(A)$. In this case $A_{i} \lambda_{i} I_{i}$ is quasi nilpotent except for possibly one $i$.

\section{REFERENCES}

1. Tsuyoshi Andô, On hyponormal operators, Proc. Amer. Math. Soc. 14 (1963), 290-291. MR 26 \#2884.

2. William B. Arveson and Jacob Feldman, $A$ note on invariant subspaces, Michigan Math. J. 15 (1968), 61-64.

3. S. Berberian, Introduction to Hilbert space, University Texts in the Mathematical Sciences, Oxford Univ. Press, New York, 1961. MR 25 \#1424.

4. F. E. Browder and W. V. Petryshyn, The solution by iteration of linear functional equations in Banach spaces, Bull. Amer. Math. Soc. 72 (1966), 566-570. MR 32 \#8155a.

5. Frank Gilfeather, Asymptotic convergence of operators in Hilbert space, Proc. Amer. Math. Soc. 22 (1969), 69-76.

6. - . The structure of non-unitary operators and their asymptotic behavior, Thesis, University of California, Irvine, Calif., 1969.

7. T. A. Gillespie, An invariant subspace theorem of J. Feldman, Pacific J. Math. 26 (1968), 67-72. MR 37 \#6787.

8. P. R. Halmos, Invariant subspaces of polynomially compact operators, Pacific J. Math. 16 (1966), 433-437. MR 33 \#1725.

9. Edwin Hewitt and Karl Stromberg, Real and abstract analysis. A modern treatment of the theory of functions of a real variable, Springer-Verlag, New York, 1965. MR $32 \# 5826$. 
10. Joseph G. Stampfli, Hyponormal operators, Pacific J. Math. 12 (1962), 14531458. MR $26 \# 6772$.

11. - Hyponormal operators and spectral density, Trans. Amer. Math. Soc. 117 (1965), 469-476. MR 30 \#3375.

12. B. Sz.-Nagy and C. Foinș, Analyse harmonique des opérateurs de le espace de Hilbert, Masson, Paris et Akadémiai Kiadó, Budapest, 1967. MR 37 \#778.

University of California, Irvine, California 92664 and

Indiana University, Bloomington, Indiana 47401 